\title{
Using Geospatial Intelligence to Promote Precautionary Behavior During the COVID-19 Pandemic: Development and Validation of a Customized Messaging Campaign in Saudi Arabia
}

\author{
Mayda Alrige ( $\nabla$ malraegi@kau.edu.sa ) \\ King Abdulaziz University https://orcid.org/0000-0002-1315-7053 \\ Hind Bitar \\ King Abdulaziz University \\ Maram Meccawy \\ King Abdulaziz University \\ Balakrishnan Mullachery \\ Claremont Graduate University
}

\section{Research Article}

Keywords: COVID-19, mHealth, customized messages, precautionary health behavior, Fogg Behavior Model, GeoAl, Space Time Cube

Posted Date: May 6th, 2021

DOI: https://doi.org/10.21203/rs.3.rs-471552/v1

License: (9) This work is licensed under a Creative Commons Attribution 4.0 International License. Read Full License 


\section{Abstract}

\section{Background}

Designing a health promotion campaign is never an easy task, especially during a pandemic of a highly infectious disease such as COVID-19. In Saudi Arabia, many attempts have been made to raise public awareness about COVID-19 infection and precautionary health measures. However, most of the health information delivered through the national dashboard and the COVID-19 awareness campaigns are generic and do not necessarily make the impact needed to be seen on individuals' behavior.

\section{Objective}

The objective of this study is to build and validate a customized messaging campaign to promote precautionary health behavior in individuals during the COVID-19 pandemic. Customization is realized by utilizing a geospatial artificial intelligence technique called the space-time cube (STC) technique.

\section{Methods}

This research was conducted in two sequential phases. In phase 1, an initial library of 32 messages was developed and validated based on Fogg behavior model for behavior change. In phase 2, we applied STC as a geospatial artificial intelligence technique to create a local map for one city with three different profiles representing the city districts. The model was built using COVID-19 real clinical data. The messages created in phase 1 is to be customized for residents in each city profile separately.

Results

n phase 1, 32 messages were developed based on resources from the World Health Organization and the Ministry of Health in Saudi Arabia. The enumerated content validity of the messages was established through the utilization of the content validity index (CVI). Thirteen professional health care workers $(n=13)$ evaluated the content of the message libraries in Arabic and English. Thirty-two messages were found to have acceptable content validity $(I-C V I=0.87)$. In phase 2 , the geospatial intelligence technique we applied showed three zones for the districts of Jeddah city: one for high infection, another for moderate infection, and the third for low infection. Combining the results from the first and second phases, a customized awareness campaign was shaped for the city of Jeddah.

\section{Conclusions}

This awareness campaign can be utilized by health authorities to raise individuals' awareness about the precautionary measures that should be taken based on the infection level of their district, and hence help in reducing the number of positive cases in the city of Jeddah.

\section{Introduction}




\subsection{Background}

Designing a health promotion campaign is never an easy task. ${ }^{1}$ This challenge becomes even more significant when the campaign takes place during a pandemic of a highly contagious disease or in the middle of a viral outbreak. ${ }^{2}$ During these critical periods, the public's precautionary measures and their compliance with health authorities' instructions could make all the difference in containing the virus. Otherwise, the virus may continue to propagate in the community and could eventually bring the countries health system to its knees. This is the current situation with the new coronavirus, known as COVID-19. After many months of lockdown, and before the world goes into a deep economic recession, many countries are choosing to reopen their public places and resume business as normal. However, with no verified medicine, a slow vaccination process, and the continued emergence of new COVID-19 variants, health authorities worldwide have to continue relying on lowering the numbers of infected individuals through precautionary measures. This can be achieved by increasing the public's awareness and compliance. Hence, an effective health awareness campaign must be put in place.

Depending on the most influential mechanisms, many health campaigns choose to apply the communication processes of awareness, instruction, and persuasion to steer the audience, via messages, toward desired behavioral changes. The content of these messages would vary through different points in the campaign. ${ }^{3}$ Awareness messaging aims at "informing people what to do, specifying who should do it, and cuing them about when and where it should be done."3 Thus it appropriate that the messages are easy to understand, and their recommended actions be simple to perform. Awareness health campaigns want to encourage the population, or segments of it, to seek more information. On the other hand, detailed instruction is only suitable if the required action is complex and where the audience needs a detailed blueprint to deal with certain health situations. This type of instruction could be seen as training in the form of a message. Meanwhile, persuasion messaging is intended more for high-involvement health practices. ${ }^{4}$

The research presented in this study focuses on the awareness process, as it is more suitable at the current level of the COVID-19 health campaign in Saudi Arabia. The targeted messages are of a simple nature and require simple, doable actions. These criteria are aligned with the previously mentioned objectives of an awareness campaign. According to Fogg Behavior Model (FBM), an individual will act and change his or her behavior if his or her motivation and ability are high, and a trigger is present at the same time.

Saudi Arabia, like other hard-hit countries, has taken stringent measures to control the rapid spread of the COVID-19 pandemic. The Saudi Arabian Ministry of Health $(\mathrm{MOH})$ has conducted an intensive awareness campaign, communicated via its website, television, and various social media channels, to equip individuals with facts and precautionary measures surrounding COVID-19. Moreover, the MOH has provided a geographic information system (GIS) dashboard ${ }^{5}$ featuring an interactive map of COVID-19related data on a national and a municipal level (see Figure 1). 
Although this map is useful, as it focuses on each region as well as each city, it does not provide details about areas within each city. The city's overall data about COVID-19 might be sufficient for smaller towns and cities, where the population is around 100,000 or less, but may prove insufficient for larger cities such as Jeddah.

Also, the Ministry of Health has provided a number of Arabic and English mobile applications to deal with the virus outbreak. Among those are Tabaud (meaning distancing in Arabic), which can alert a person if he or she has been exposed to someone who has reported having COVID-19 and Tetamman (meaning rest-assured in Arabic), which aims to reinforce the commitment of all persons directed to self-isolation and follow-. From a technical perspective, the apps work fine; however, they are only of value if given real data. Also, since downloading and activating them is optional many individuals might choose not to do so for many reasons. These reasons include privacy concerns, as the idea of being tracked by one's government might seem too intrusive to some.

The $\mathrm{MOH}$ has also launched a COVID-19 pandemic health awareness campaign through different media channels, including web platforms and social networks. The intent of this campaign is to educate the public about the highly contagious virus. These efforts include sending text messages to mobile phones, prompting the adoption of healthy habits, and encouraging the public to take appropriate virus prevention measures. A few examples are shown in Figure 2.

Designing campaigns customized to individuals or groups of individuals has been widely studied in health communication research. ${ }^{6}$ Customized text-based intervention was found to be significantly associated to greater intervention efficacy. ${ }^{7}$ For example, Pfammatter et al ${ }^{8}$ examined the impact of sending messages to patients with diabetes to increase exercise and improve fruit, fat, and vegetable intake. The participants were selected randomly and assigned to an intervention group or a control group. Participants in the intervention group who received the text messages demonstrated better improvement in health behavior compared to participants in the control group, who did not receive any text messages. ${ }^{8}$ According to Horner et al, ${ }^{9}$ using this type of intervention can assist in increasing the physical activities of patients with type 2 diabetes. Horner et al ${ }^{9}$ designed the study as a two-parallel group randomized controlled trial, where the intervention group received text messaging twice a day. The participants satisfaction were very high with the more than $90 \%$ of them stating that they will recommend these messages program to a friend. The participants of this study described these messages as motivational, informational and educational. However, while the participants were satisfied with the text messaging, they suggested that these messages are better be personalized. ${ }^{9}$ In another study, text messaging intervention was used to increase and maintain Saudi women's ability to educate themselves about HPV, enhancing their knowledge about HPV and cervical cancer. Around 34 Saudi women were recruited using convenience and snowball sampling techniques. The authors applied a single group, pre- and postintervention method. The results showed a statistically significant difference in self-efficacy before and after receiving the text-messages. ${ }^{10}$ 
There are two methods for customized health messages: targeting and tailoring. Message targeting uses customized messages based on the characteristics of a subgroup of an overall population; for example, they would target people with similar lifestyle traits, such as fresh college graduates starting their careers or people who live in a certain geographical area. Message tailoring fits messages to individual characteristics, such as personality factors, ways of thinking, or coping styles. Each of these methods has its advantages; the ability to select which one to apply to a certain campaign depends on the campaign itself and the available data about the target audience. ${ }^{6}$

The population data available along with the resources, timing, and type of messages being propagated to the target audience make the message targeting method more suitable for this study. This health awareness campaign has been adapted to educate the public about simple safety measures to prevent them from being infected by COVID-19. The targeted audience for this customization would be those within a geographical location, namely, on a district level within the city of Jeddah. People within the targeted location would receive messages regarding how many infected cases had been reported in their particular area, the sources of infection (if available), or the recipients' proximity to areas where many COVID-19 cases have been reported.

\subsection{Objectives}

This research aims to design, develop, and evaluate the content of a customized awareness campaign to reinforce precautionary health behaviors of individuals during the COVID-19 pandemic. This messagebased campaign will be developed in two sequential phases. The first phase aims to design and evaluate messages by employing the Fogg behavior model (FBM). ${ }^{11}$ The FBM is a holistic model describing behavior change as the convergence of three components: motivation, ability, and trigger. These three elements are the building blocks of our campaign content. FBM was initially developed to guide the design of persuasive technology, which is technology that aims to promote a certain behavior. In this study, the model is followed to develop an awareness campaign aiming to promote precautionary health behavior using messaging intervention. The awareness campaign content is then evaluated by domain experts for its relevance to the three conceptual components of FBM. ${ }^{12,13}$

The second phase of this research applies a geospatial intelligence platform to profile the city of Jeddah. These location-aware profiles, combined with behavior-change messages built in the first phase, will be used to target the locals of each district differently. The content of this phase will be driven by statistics of confirmed COVID-19 cases and the source of the infection on a district level, both analyzed using ArcMap. ${ }^{14}$

\subsection{Theoretical Framework}

To change or promote a certain human behavior, theories of behavior change are needed. The present study is guided by FBM to build the content of the messages. The Fogg model highlights three main 
factors: motivation, ability, and trigger. To embrace a new behavior, a person must be motivated, have the ability to perform the behavior, and be prompted to do so with an effective trigger.

For example, if the target behavior is to wear a face mask while a person is outside, a person must have sufficient (high) motivation, ability, and an effective trigger. These factors must be present at the same instant for the behavior to occur, which is to wear a face mask every time this individual goes outside.

As reported by Fogg, while designing the motivation, the goal must be to move the user to a higher position in the FBM landscape, which means to increase the user's motivation. There are three core motivators: pleasure/pain, hope/fear, and social acceptance/social rejection. In this study, we will use the hope/fear motivator to build the content of the messages. The hope/fear motivation is sourced from the anticipation of something good (hope) or bad (fear) happening. For example, the hope of not being infected by COVID-19 will motivate a person to follow government safety roles and stay at home, while a person will wear a face mask to overcome the fear of getting infected by COVID-19. Another example is that a person will keep a social distance to overcome the fear built from the anticipation of getting COVID-19.

The second factor in FBM is ability. The most important condition in this factor is simplicity, since, as stated by Fogg, "Simplicity changes behaviors." 11 To increase a person's ability, the persuasive technology design must make the behavior easy to do. For example, one-click shopping on Amazon or short and easy-to-understand messages on a phone or social media account. The author defined six elements that detract from simplicity: time, money, physical effort, brain cycles, social deviance, and nonroutine. If the new or target behavior requires time to perform, it is not simple. The money element of simplicity refers to the financial requirements of the behavior; if the target behavior is costly or requires money, it is not simple, especially for users with limited monetary resources. The third element is physical effort: the new behavior must not require physical effort; otherwise, it will not be simple to do. The fourth element is brain cycles, meaning that hard thinking is required. If the target behavior requires the user to think a lot, the task is not simple. Another element is social deviance; this element describes going against the norm or breaking society's rules. If performing a new behavior will break certain rules, it is not simple. Finally, the last element is non-routine, indicating that the new behavior is not from a user's routine. If a person is seeking a behavior that is not from his or her routine, he or she may think it is not simple. ${ }^{11}$ An example of a new behavior required during the COVID-19 health crisis is wearing a face mask. Performing this behavior will not take time, especially if a person follows some tips, such as putting the mask near the entrance. A face mask is not expensive. Also, there is no effort required to wear the mask. The user will not need efforted thinking to wear the mask. Furthermore, this behavior is not currently against society's rules, as it is one of the safety rules. Finally, non-routine: yes, wearing a face mask is not a routine, but it will be. Therefore, in most societies, people must consider this behavior as part of their routine while going outside their home.

The third factor is trigger. Fogg defines trigger as "something that tells people to perform a behavior now." ${ }^{11}$ There are three types of triggers: facilitators, signals, and sparks. Facilitator triggers are used if 
the users have high motivation but lack ability. This type of trigger can be expressed in text, video, graphics, and by other means. They are effective if they focus on simplifying the target behavior, for example, by telling users that performing a certain behavior is easy, does not take time, and is not costly. The second trigger type is signals. These triggers are a good fit if the users are high in motivation and ability, but just need a reminder. For example, a morning message to remind people to wear their face mask if they are going outside is a signal trigger. Finally, sparks triggers are used if the person lacks the motivation to perform the target behavior. The technology that will be used here must focus on the motivational element, for instance, texts to highlight the fear of catching COVID-19, or videos that highlight hope of not getting COVID-19. The most important element of these triggers is that they are "presented to the user at a moment when they can take action."11

\section{Methods And Materials}

The research approach was conducted in two sequential phases, as shown in Figure 3. The first phase aimed to develop a library of awareness campaign messages based on FBM. The authors built and evaluated three message groups following the Alismail and Olfam research method of validating different messaging libraries. ${ }^{15}$ These messaging groups highlighted the three main factors underlying FBM: motivation, ability, and trigger. The second phase applied geospatial artificial intelligence (GeoAl) to better visualize the infection level of each district within the city. This specific location-aware information is to customize a messaging campaign distinctly targeting the locals of each district. The rest of this section will elaborate on these phases sequentially.

\subsection{Developing the FBM-Based Messaging Campaign}

The authors designed and developed two main libraries in two different languages: Arabic and English. These messaging libraries were designed and developed based on FBM's main factors: motivation, ability, and trigger. We developed 30 messages using trusted sources, such as the World Health Organization and the Saudi $\mathrm{MOH}$. The messages can be categorized into three main groups: the facilitator-trigger (Ability-Simplicity) group, the signal-trigger (Reminder) group, and the sparks-trigger (Hope/Fear motivator) group, as shown in Figure 4.

All 30 messages were designed to address all the factors of FBM that need to be changed, increased, or occur. We created 10 messages for each of the three groups, as can be seen in Figure 4 . The following are explanations of each of the messaging groups:

1. Ability-Simplicity Messaging Group: These messages target individuals with low abilities and high motivations. The messages are designed to simplify the new actions.

2. Hope/Fear Motivator Messaging Group: These messages target individuals with high abilities and low motivations. The messages include the motivational elements hope and fear: fear of being infected with COVID-19 and hope of being healthy and not being infected. 
3. Reminder Messaging Group: These messages target individuals with high abilities and motivations. The messages are reminders to perform the actions.

The initial libraries, written in Arabic and English, include 30 messages for each library. All the messages are text-based except for a single video message. The research team generated these messages in iterative processes. First, we searched and reviewed trusted sources, such as the World Health Organization, to obtain significant facts and statistics about COVID-19 and simple precautions the public could follow to fight the spread of the disease. Then, we started to write the messages using the FBM factors in Arabic. After that, the translation process was implemented based on Brislin's techniques for cross-cultural research. ${ }^{16} \mathrm{He}$ recommends the back-translation and bilingual techniques (outlined in Textbox 1).

Textbox 1. Translation Process Steps

\section{A. Back-Translation Technique}

1. Write all messaging in Arabic,

2. Translate all messages into English,

3. Ask an expert who is a bilingual to back-translate all the English messages into Arabic,

4. Ensure that all messages reflect the exact meaning and context.

\section{B. Bilingual Technique}

1. Ask another bilingual expert to review the messages in both languages to ensure the validity of the messages,

2. One of the bilingual authors in this research study reviews the messages one more time.

\section{Analysis}

The campaign messages were created to motivate, to trigger, or to demonstrate one's ability to adhere to precautionary measures. Once the message libraries were crafted in both languages, they became the target for content validation by domain experts. The domain experts chosen for this research were professional healthcare workers. They evaluated the content of the messages with respect to the concepts of motivation, ability, and trigger.

To evaluate the content validity of the campaign messages, we utilized the content validity index (CVI). ${ }^{12}$ The CVI for each item (termed the I-CVI) is determined by the number of experts giving a rating of 3 (quite relevant) or 4 (highly relevant) divided by the total number of experts. An I-CVI score of 1.00 indicates $100 \%$ agreement among the experts, while messages with a low I-CVI score (for example $<1.00$ ) should either be modified or deleted for clarity. When there are more than five experts, there can be a modest amount of disagreement; for example, when there are six experts, the I-CVI must be at least 0.83 , reflecting one disagreement. Another index used to assess message content validity is the scale content 
validity index (S-CVI), which considers the validity of all the messages together. The S-CVI can be calculated by averaging the I-CVI scores. The S-CVI/Ave function computes the I-CVI for each item on the scale, and then calculates the average I-CVI across all the items. The steps of content validation are outlined in Textbox 2.

Thirteen medical experts $(\mathrm{N}=13)$ completed the survey for the three sets of messages. Of these experts, there were 9 nurses, 2 dentists, a physician, and a community medicine specialistc. The message library consisted of 30 messages divided into three sets. The first set of messages is to motivate the audience. The second set of messages promotes one's ability to adhere to precautionary measures. The third set of messages is to trigger health behavior using reminders.

Textbox 2. Content Validation Steps to Calculate I-CVI

1- Define the three concepts behind the Fogg behavior model and measure each item's relevance to them. For each item(message) in the library, experts rate the relevance of each item on a 4-point scale. (1: not relevant, 2: somewhat relevant, 3: quite relevant, 4: highly relevant).

2- For each item, the I-CVI is computed as the number of experts giving a rating of either 3 or 4 , divided by the number of experts.

3- If there are 5 or less experts, the I-CVI should be 1.00 to be acceptable. If number of experts exceeds $5, \mathrm{l}-\mathrm{CVI}$ should be at least 0.83 .

4- If the I-CVI for one particular item is less than 0.83 , the item must be deleted or be modified and go for another round of revision.

5- Once I-CVI is calculated for each item, then S-CVI should be calculated.

6- S-CVI is be obtained by averaging the I-CVIs.

7- The minimum acceptable level for S-CVI/Avg is 0.80

\subsection{Applying Geospatial Analysis to Profile Jeddah City Districts}

The second phase of this research aimed to geographically customize the awareness messages developed in the first phase. We utilized COVID-19 clinical data in the city of Jeddah, Saudi Arabia. To target the locals of each district separately, we visualized the COVID-19 clinical data on a local map using geospatial artificial intelligence technology. ${ }^{17}$ This step will help in both build a profile of each district in terms of COVID-19 infection levels and target the awareness messages for each district independently. This contextual information will be used to customize the messages for each district's locals. The technical work of applying geospatial artificial intelligence in phase 2 has three steps, as seen in Figure 5.

\subsubsection{Data Collection and Preparation}

Data was collected from governmental (public) health providers in Jeddah. Official approval for data collection was obtained by the Saudi $\mathrm{MOH}$ authorities in Jeddah, which named three specific hospitals in the city to be accessed for the purposes of this research (Consent is in Appendix 1 ). All team members 
obtained a certificate for good clinical practice prior to the granting of data access. One hospital, King Fahad General Hospital, fully collaborated and provided the requested clinical data, which included on the individual level: age, gender, address, patient status, and source of transmission. In total, the records of 2750 individuals who had tested positive for COVID-19 between March 25 and June 25, 2020 were obtained from this hospital. Although the data was provided, it required further pre-processing and manipulation before being inserted into the GIS application.

The data provided by health authorities required pre-processing and manipulation before being inserted into the GIS application (ArcGIS). For example, some clinical data were split between two different files; one contained the patients' personal information while the other held their district addresses. Hence, some records had to be matched manually using the patient's record ID. After the matching process was performed and any records with missing or incomplete data were eliminated, the total number of available records to be used for this research was 477 medical records.

\subsubsection{Creating a Local GIS Map of COVID-19 for Jeddah by District}

The dataset was processed and analyzed using an ArcGIS application. ArcGIS is an online cloud-based mapping, analysis, and data storage system that can be used to create, share, and manage maps, scenes, layers, apps, and other geographic content. ${ }^{14}$ Data was inserted into ArcGIS to create a local map of Jeddah districts showing confirmed cases of COVID-19. Most of the source raw data file received was missing the patients' spatial and address information. The geospatial technique known as space time cube (STC) analysis was utilized to target specific geolocation areas with high infection. The clinical data of COVID-19 cases in each district, obtained from three major health care providers, was used to feed the STC model.

STC allows visualization of spatio-temporal data in 2D or 3D, analyses distribution of the data, and temporal pattern analysis in the context of space and time. Each bin represents a collection of the event at a specific time for a specific location. The lowest bin is the oldest, and the highest bin is the latest event that represents a time series. The horizontal slicing of the cube represents the time-slicing of the condition of all the events in that period. In the context of COVID-19 cases, the lowest bins represent the earliest positive cases found, while the highest bins represent the most up-to-date positive cases confirmed.

\subsubsection{User (District) Modeling}

To create the customized awareness campaign, user modeling needs to take place. User modeling is a human-computer interaction used to illustrate the process of construction and modification of a certain conceptual understanding ${ }^{19} \cdot$ In our research, STC analysis was used to profile the city of Jeddah based on the infection levels of each district. 


\section{Results}

\subsection{Evaluating the FBM-Based Messaging Campaign}

We validated the content of the messages in two rounds; in round 1, the S-CVI/Ave was 0.94 , and the I-CVI was above the threshold value ( 0.83$)$ for 28 out of 30 messages. This indicates that 28 out of 30 messages were found relevant to FBM theoretical concepts. Only 2 messages were found irrelevant by experts: one motivation message and one ability message $(I-C V I=0.78)$. These messages were deleted and replaced by another 4 messages in a second iteration. These 4 messages were evaluated by 8 domain experts. The content validity for the added messages was much higher (I-CVI=0.87). Thus, these four messages were included in the awareness messaging campaign, yielding a total of 32 messages (see Appendix 2).

\subsection{Profiling City of Jeddah Geospatially}

In phase 2, the geospatial technique known as space time cube (STC) analysis was used to profile the city of Jeddah based on the infection levels of each district. The clinical data of COVID-19 cases in each district, obtained from three major health care providers, was used to feed the STC model. Data collection and preparation had been performed and explained in the methods section. The two remaining technical steps taken in this phase are outlined below.

\subsubsection{The resulted Local GIS Map of COVID-19 for Jeddah by District}

The raw data were manually geocoded using the city and district name via the ArcGIS world geocoding process. The total number oAf geocoded cases used in the analysis was 69. With data about COVID-19positive patients alongside the districts in which they reside, the map of Jeddah (Figure 6) shows which district has the highest number of recorded infected cases, and hence residents will be alerted about how "close" they are to the infected area. For example, Al-Jameaah district was the highest with a total of 10 cases, followed by Madaan Al-Fahad district with 7 cases from June $18^{\text {th }}$ to June $30^{\text {th }}$.

In Jeddah city neighborhoods, the number of cases is distinct compared to the neighboring districts. The darker cube represents more activity on that particular day. In this study, an STC was created by aggregating COVID-19 cases within a $5 \mathrm{~km}$ radius, with each bin representing an aggregated case-load for a single day. The bins are rendered from the oldest to the newest time series data. The COVID-19 case trends are visualized using a range of bin colors, as shown in the legend for Figure 6.

\subsubsection{The resulted User (Districts) Models}


In the context of this research, user modeling was performed to give individuals a meaningful and customized message about the status of the coronavirus pandemic in their district. Modelling is according to two levels, described as follows:

Level 1: District Level. In this process, each district or group of districts with similar characteristics, must be modeled and given a unique user profile. Districts were divided into three main zones (profiles) in terms of how they are affected by the pandemic: high (red), medium (orange), and low (yellow). The levels that resulted from the performed analysis using STC can be seen in Figure 7. The red color indicates that the confirmed cases number is high $(>=5)$, the orange color indicates a moderate number of confirmed cases (3-4), and the yellow color indicates that the number of daily cases is very low $(<=2)$. Based on these profiled districts, the messaging campaign should target each district differently.

For example, awareness messages can be sent daily to people who live in the red zone, such as AlJameaah district. For the orange zone, the awareness messages can be sent every other day. Finally, residents who live in the yellow zone can receive the message twice a week, since the infection level is very low.

Level 2: Group Level. In this process, individuals residing in those districts were grouped (profiled) further according to gender and age. This assists in meeting personal needs to influence one's behaviors. ${ }^{6}$ Looking at the patients' characteristics for the Al- Jameaah district from June $18^{\text {th }}$ to July $1^{\text {st }}$ (see Table1), there are 3 female patients aged 29 to 32 . Hence, the message content can be targeted to this demographic according to the activities they do and places they visit most often. In addition, Table 3 shows that the most infected cases are male between the ages of 23 and 55 . The awareness message can be tailored based on these two parameters, and more message groups can be generated.

\section{Discussion}

The strength of the proposed awareness messaging campaign stems from two aspects. The first aspect is that the campaign is guided by the behavior change model, FBM. To the best of our knowledge, this is the first time that FBM is being used in public health informatics to develop an awareness campaign and raise public awareness about precautionary measures during a pandemic. Following such a theoretical model when building and designing any health intervention is valuable and recommended by social and behavioral research. ${ }^{22}$ Promoting a certain health behavior, such as, in this case, a precautionary health behavior for individuals in a specific geographic location, is sufficient and valuable if the messaging campaign content that is built is based on solid behavior change theory.

The second aspect is that the messaging awareness campaign can be customized to the district level to target highly infected areas. Customization or audience segmentation has proved useful for health communication messages. ${ }^{6}$ Health educators recognize the importance of audience segmentation as a means of reaching the neediest members of a population. This research approach toward building a customized health campaign will provide locals in the city of Jeddah with a better understanding and 
awareness of their approximate exposure to the virus without jeopardizing their privacy. This approach is less invasive than tracking mobile applications, where some individuals might hesitate to install and use them due to privacy concerns. Individuals may be more likely to apply the health tips given in the awareness message if the message is equipped with more contextual information from where the individual lives. Currently, the Saudi $\mathrm{MOH}$ has utilized information technology systems, tools, and applications to a good extent during the COVID-19 pandemic, including the use of GIS maps, locationbased services via mobile applications, and mobile text messages, among others. However, there is still room for improvement. This research is one attempt to achieve such a goal. This research utilized a geospatial artificial intelligence technique, the space-time cube, to create a local map that provides city district data regarding COVID-19 cases, going one level beyond the national GIS dashboard map by $\mathrm{MOH}$ that shows the data at national and city levels. We used health data from the highly populated city of Jeddah as a case study to build a program for increasing city residents' awareness by sending customized messages, focusing initially on the district in which people reside (first level) and age and gender (second level).

\section{Limitations and Directions for Future Work}

One of the main limitations in this research was accessing the data set about local patients in Jeddah. The first three authors received online training and became certified in Good Clinical Practice, to ensure data privacy of medical records, and therefore were granted access to data from three major general hospitals from the $\mathrm{MOH}$. However, the procedure to obtain patient data from these hospitals was complex. Data collection took place in the summer of 2020, and while the lockdown in Saudi Arabia had just been lifted, COVID-19 precautionary measures were still very strict. One hospital, for example, only allowed access to the data from within its premises, and the hospital visitation for non-patients and nonstaff was limited. The required data itself was not located in one medical record system, but rather, was scattered across a number of legacy systems that differed from one hospital to another. Moreover, some records were missing crucial personal information, such as the patient's address. As a result, the project (which received university funds for a maximum of nine months) was delayed for 2 to 3 months, and the data sample size was limited in terms of the number of records as well as the period in which the data could be collected. Ideally, the authors would have preferred continuous access to many records over an extended period to create a more reliable and dynamic map and demonstrate district profiles in real-time or near real-time.

Another limitation is not being able to send the customized messages and measure the impact of awareness level due to logistic barriers resulting from social distancing rules. In future endeavors,

a case study can be conducted to send and measure the impact of customized messages focusing on at least three districts, one from each of the red, orange, and yellow zones. On the first level of customization, depending on the level of the district in which one lives, an individual will be targeted more or less frequently by the awareness campaign messages, as will everyone else who has a registered 
address in the same district. For example, if one lives in Al Sharafiyhay district, which has been assigned as a high-infection district (represented as red on the map), one will receive the number of messages that match this profile. If one lives in Al Naeem District, which has a low level of infection (marked yellow on the map), he/she will get a lower number of messages according to their district profile, and so forth. On the second level of customization, individuals will receive certain targeted messages from our COVID-19 awareness campaign library (see Appendix 2), depending on their profile of location, gender, and age. Multi-variant analysis can be used to measure the impact of the messaging campaign on the COVID-19 infection level.

Tailoring can add another level of customization. The campaign message can be tailored to individuals based on their personal characteristics; in our research context, it would be based on their motivation and ability to adhere to the precautionary measure. Following this direction, a one-group, pre- and postintervention study can be used to measure the participants' motivation and ability levels before and after the campaign. For this purpose, Agha et al's instrument ${ }^{18}$ can be used to place each participant in the appropriate group profile. A pilot test can be conducted to account for any adjustments and changes to the instrument. Subsequently, the data can be analyzed using a paired sample $t$ test to see the effectiveness of the messages. The following steps will be conducted: (1) measure each participant's ability and motivation (pre-intervention), (2) distribute the participants into different groups based on the results, (3) send each participant awareness messages from the library, (4) remeasure each participant's ability and motivation (post-intervention), and (5) analyze the data using paired sample $t$ test. If the participants' ability and motivation have increased and the number of COVID-19 cases have decreased in that specific district, the awareness messaging campaign may be considered to have an acceptable level of effectiveness. This local infection map can be set up to send awareness messages to locals of a district with high infection as well as to other areas, with a variable number of messages that will be sent on a weekly basis, as explained in a previous section. Moreover, the resulting local map will be linked to a messaging system that, after recruiting the participants and registering them, will automatically send the survey to measure participant ability and motivation. Finally, the system will allocate participants into different groups in order to start sending the awareness messages from the library.

Furthermore, this research approach of building, validating, and customizing the awareness campaign can be followed to design awareness campaigns during the outbreak of any other infectious diseases. Certain parameters in the approach would have to be changed based on the nature of the virus. For instance, respiratory system viruses such as SARS-CoV-2 spread primarily through droplets generated when an infected person coughs or sneezes, or through droplets of saliva or discharge from the nose. This kind of respiratory virus requires certain precautionary measures. Parameters that might have to be changed would include the precautionary measures described in the messages, the types of clinical data that must be aggregated in the localized map, and the city represented by the map.

Finally, Hot analysis has been widely used in spatial epidemiology to target audience-specific segments. ${ }^{18}$ Health authorities in each city can always utilize the map to send more targeted or tailored messages to the public as part of an effective awareness campaign. STC analysis can be used as real- 
time analysis. As more positive cases occur, more bins will be added with an appropriate infection level color. Health authorities in each city can use the STC local map to generate awareness campaigns for each district differently.

\section{Conclusions}

This study leverages geospatial artificial intelligence to profile the city of Jeddah. Jeddah city has more than 83 districts spread across a $1600 \mathrm{~km}^{2}$ area. ${ }^{21}$ By using $\mathrm{MOH}$ healthcare provider data to create a map of the city in which positive COVID-19 cases are represented according to district, a two-fold solution occurs: The map allows people to know (1) if they have been around areas where active and positive cases have been recorded and must take necessary steps to prevent being infected with COVID-19, and (2) that they should avoid visiting those areas if possible, based on the customized messages. The STC technique showed three profiles for the districts of Jeddah city: one for high infection, another for moderate infection, and the third for low infection. This map can be used by health authorities to initiate the awareness messaging campaign we crafted in the first phase. The residents of these profiled districts can be targeted separately by this campaign based on the COVID-19 infection levels of their specific geographic locations as well other shared characteristics.

\section{Abbreviations}

$\mathrm{MOH}$ : Ministry of Health

FBM: Fogg behavior model

GeoAl: Geospatial artificial intelligence

GIS: geographic information system

STC: space time cube

\section{Declarations}

\section{Ethics approval and consent to participate}

Consent from Ministry of Health to collect real clinical data from three major health care providers is available up

\section{Consent for publication}

Available upon request.

\section{Availability of data and materials}




\section{Competing interests}

Not declared

\section{Funding}

This project is funded by the Deanship of Scientific Research (DSR) at King Abdulaziz University, Jeddah, under grant no. GCV19-11-1441. The authors, therefore, acknowledge and thank DSR for their technical and financial support.

\section{Authors' contributions}

- Hind Bitar was responsible of building the messaging campaign following Fogg Behavior Model three concepts: motivation, ability and trigger.

- Mayda Alrige was the idea owner and was in charge of evaluating the content validity of the messages library using Content Validity index (CVI).

- Balakrishnan Mullacherry was in charge of applying the Space Time Cube using Covid19 clinical data to profile the city of Jeddah.

- Maram Meccawai was in charge of profiling the city of Jeddah and refining the content of the messages libraries and translating them into English.

\section{Acknowledgements}

We would like to thank Dr. Marwa Afif from King Fahad General Hospital for her constant support while processing the COVID-19 clinical data. We would also like to thank Mrs. Raghad Gharbawi and Mrs. Samar Alahmadi for their contribution to this research.

\section{Authors' information}

Mayda A. Alrige is an Assistant Professor of Information Systems in King Abdulaziz University. Her research interests revolve around artificial intelligence and knowledge management. Recently, her work is focused on utilizing fuzzy-logic and multi agent systems for nutrition education and diet recommendation. She is particularly interested in the utilization of ambient intelligence and ubiquitous technologies for smart healthcare sector and smart cities. She has a bachelo's degree (BSc) in Computer Science from KAU (2009), master's degree (MSc) in Information Systems from College of Engineering and Information Technology at University of Maryland- BC (2013). During her masters, she became an active member at the Healthcare Informatics and Technologies Lab and conducted her master thesis in privacy preserving integration of publicly available health data. Mayada received her PhD in Information Systems 
and Technology from Claremont Graduate University (2018). For her PhD dissertation, she developed an intelligent nutrient profiling engine, a rule-based system, to educate type-2 diabetics about the nutrient content of their food and recommend customized diet. This research project was conducted in collaboration with the diabetes treatment center at Loma Linda University Diabetes Treatment Center, LLU. More about her work can be found in her Google Scholar Web Page

HIND BITAR is an assistant professor at the Information Systems Department, FCIT, King Abdulaziz University in Jeddah, Saudi Arabia. She obtained her undergraduate degree in Computer Science (Umm Al-qura university, SA, 2008). She earned her master degree in Information Systems from University of Maryland Baltimore County (UMBC), US in 2014. Dr. Bitar received her Ph.D. in Information Systems and Technology, health informatics (Claremont Graduate University (CGU), USA, 2018). Dr. Bitar reviewed many scientific research papers for several journals and conferences, such as JMIR, JAMIA and HICSS. Bitar has made some scholarly contributions in the field of health and energy informatics, as well as geoinformatics. Also, she is currently working on several research projects that have been funded by King Abdulaziz University in the health-related domain.

MARAM MECCAWY is an Associate Professor at the Department of Information Systems, Faculty of Computer Science and Information Technology at King Abdulaziz University (KAU), Saudi Arabia. She received her Ph.D. from the University of Nottingham in the UK. After her graduation, she spent a few years working in the industry before joining KAU as an assistant professor in 2014. Dr. Meccawy research interests include Adaptive Educational Hypermedia, User Modelling, LMS, MOOCs, and Teaching Programming to children

BALAKRISHNAN MULLACHERY received a B.S. degree in Physics from Calicut University, Kerala, India, MS in Geophysics from Andhra University, India, and second MS in Information Science and Technology (IST) with Specialization in Data Science from Claremont Graduate University (CGU), California. He is currently working toward a Ph.D. degree in Data Science. Since 2002 Mr. Mullachery has been with Environmental Systems Research Institute (Esri) as a System Engineer/Sr. GIS Consultant. The research interests are in Health informatics, Data Science, GIS, IoT, Al, GeoAl, and Image Analysis with a couple of research publications in health informatics and Geo-Analytics.

\section{References}

[1] Kelly MP, Barker M. Why is changing health-related behaviour so difficult? Public Health, 2016;136:109116. doi: 10.1016/j.puhe.2016.03.030.

[2] Randolph W, Viswanath K. Lessons learned from public health mass media campaigns: Marketing health in a crowded media world.

https://www.annualreviews.org/doi/abs/10.1146/annurev.publhealth.25.101802.123046. Published March 11, 2004. Accessed October 03, 2020.

[3] Rice RE, Atkin, CK. Public Communication Campaigns. SAGE, 2001. 
[4] Blanson Henkemans OA, van Empelen P, Paradies GL, Looije R, Neerincx MA. Lost in persuasion: A multidisciplinary approach for developing usable, effective, and reproducible persuasive technology for health promotion. In: 2015 9th International Conference on Pervasive Computing Technologies for Healthcare (PervasiveHealth); May 2015: 49-56. doi: 10.4108/icst.pervasivehealth.2015.259161.

[5] COVID 19 Dashboard: Saudi Arabia. https://covid19.moh.gov.sa/. Accessed June 30, 2020.

[6] Schmid KL, Rivers SE, Latimer AE, Salovey P. Targeting or tailoring? Maximizing resources to create effective health communications. Mark. Health Serv. 2008;28(1):32-37.

[7] Head KJ, Noar SM, lannarino NT, Grant Harrington N. Efficacy of text messaging-based interventions for health promotion: A meta-analysis. Soc. Sci. Med. 2013;97:41-48. doi:

10.1016/j.socscimed.2013.08.003.

[8] Pfammatter A. et al. mHealth intervention to improve diabetes risk behaviors in India: A prospective, parallel group cohort study. J. Med. Internet Res. 2016;18(8):e207. doi: 10.2196/jmir.5712.

[9] Horner GN, Agboola S, Jethwani K, Tan-McGrory A, Lopez L. Designing patient-centered text messaging interventions for increasing physical activity among participants with type 2 diabetes: Qualitative results from the text to move intervention. JMIR MHealth UHealth. 2017;5(4):e6666. doi: 10.2196/mhealth.6666.

[10] Bitar H, Ryan T, Alismail S. The effect of implementing an SMS messaging system to overcome the lack of transmission of HPV facts in Saudi Arabia. In the Hawaii International Conference on System Sciences, January 2020: Hawaii, United States. doi: 10.24251/HICSS.2020.598

[11]Fogg B. A behavior model for persuasive design. In Proceedings of the 4th International Conference on Persuasive Technology; April 2009. Claremont, California, USA. doi: 10.1145/1541948.1541999.

[12] Lynn MR. Determination and quantification of content validity. Nurs. Res.1986;35(6):382-386.

[13] Polit DF, Beck CT, Owen SV. Focus on research methods: Is the CVI an acceptable indicator of content validity? Res. Nurs. Health. 2007; 30(4):459-67

[14] ArcGIS.

[15] Alismail S, Olfman L. A tailored motivational messages library for a mobile health sleep behavior change support system to promote continuous positive airway pressure use among patients with obstructive sleep apnea: Development, content validation, and testing. JMIR MHealth UHealth, Aug 2020;8(8):e18793. doi: 10.2196/18793.

[16] Brislin, RW. Cross-cultural research methods. Environ. Cult. 1973. 
[17] clubdebambos, What is hotspot analysis? Geospatiality. January 21, 2016.

https://glenbambrick.com/2016/01/21/what-is-hotspot-analysis/. Accessed Aug. 12, 2020.

[18] Stopka TJ, Krawczyk C, Gradziel P, Geraghty EM. Use of spatial epidemiology and hot spot analysis to target women eligible for prenatal women, infants, and children services. Am. J. Public Health. Feb 2014;104(1):S183-S189. doi: 10.2105/AJPH.2013.301769.

[19] Fischer G. User modeling in human-computer interaction. User Model. User-Adapt. Interact. March 2001;11,(1):65-86. doi: 10.1023/A:1011145532042.

[20]Brusilovsky P. Methods and techniques of adaptive hypermedia. In Adaptive Hypertext and Hypermedia, Brusilovsky, P, Kobsa, A, Vassileva, J, Eds. Dordrecht: Springer Netherlands; 1998:1-43.

[21] Jedah Municipality Portal. https://www.jeddah.gov.sa/index.php. Accessed December 21, 2020.

[22] Luca NR, Suggs SL. Theory and model use in social marketing health interventions. $J$ Health Commun. 2013;18(1):20-40.

\section{Tables}

\section{Table 1. Al Jameaah District Patients' Characteristics}

\begin{tabular}{|c|c|c|c|c|c|}
\hline Isolation Date & City & District & $\Delta$ & Age & Gender \\
\hline $6 / 18 / 2020$ & Jeddah & AL JAMEAAH & & 32 & FEMALE \\
\hline $6 / 19 / 2020$ & Jeddah & AL JAMEAAH & & 23 & MALE \\
\hline $6 / 20 / 2020$ & Jeddah & AL JAMEAAH & & 43 & MALE \\
\hline $6 / 21 / 2020$ & Jeddah & AL JAMEAAH & & 36 & MALE \\
\hline $6 / 23 / 2020$ & Jeddah & AL JAMEAAH & & 30 & FEMALE \\
\hline $6 / 27 / 2020$ & Jeddah & AL JAMEAAH & & 29 & FEMALE \\
\hline $6 / 27 / 2020$ & Jeddah & AL JAMEAAH & & 55 & MALE \\
\hline $6 / 28 / 2020$ & Jeddah & AL JAMEAAH & & 48 & MALE \\
\hline $6 / 30 / 2020$ & Jeddah & AL JAMEAAH & & 38 & MALE \\
\hline 7/1/2020 & Jeddah & AL JAMEAAH & & 55 & MALE \\
\hline
\end{tabular}

\section{Figures}




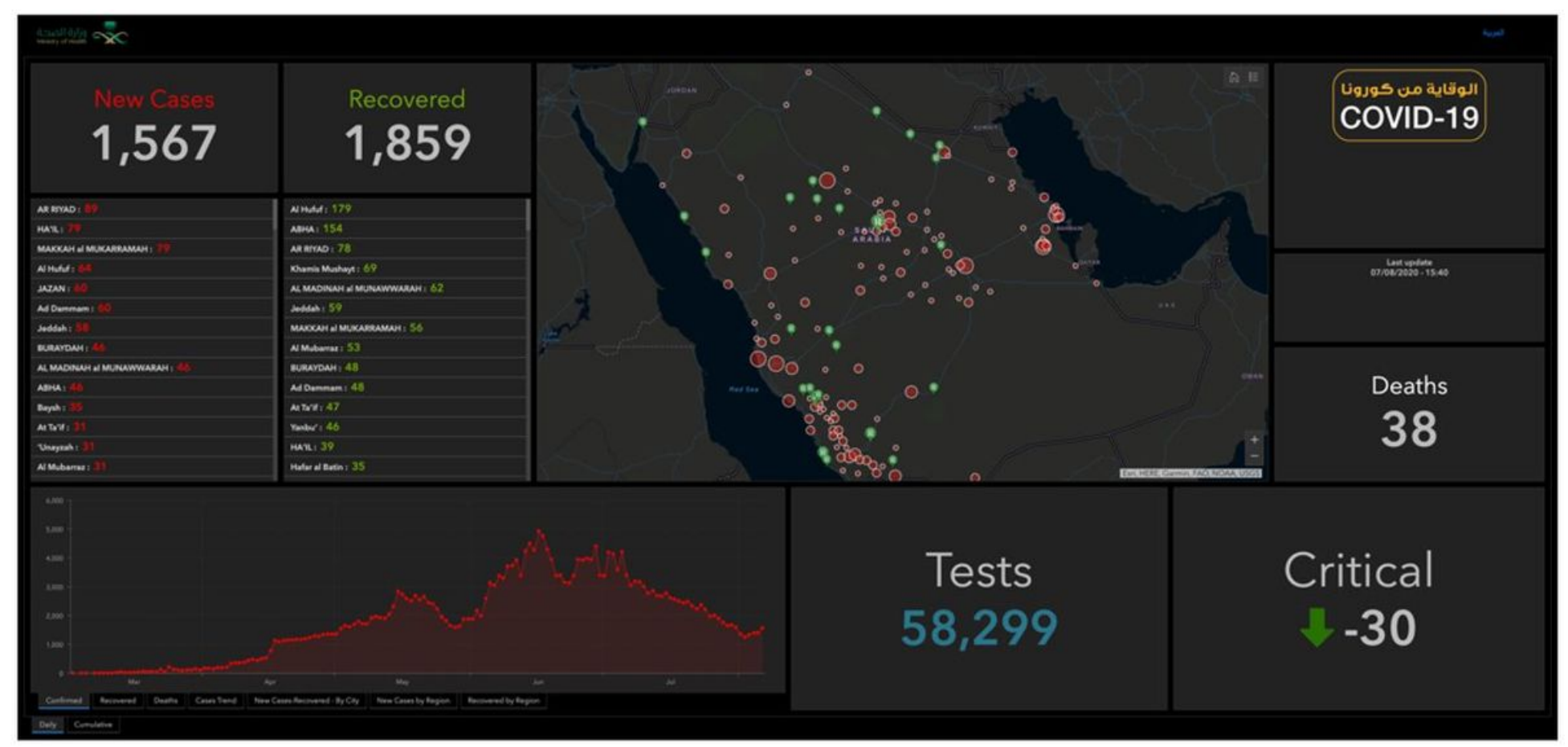

\section{Figure 1}

\section{MOH Dashboard}

\begin{tabular}{|c|c|c|}
\hline Fri, 3 Apr, 5:34 PM & Sat, 20 Jun, 2:15 PM & Fri, 17 Jul, 10:59 AM \\
\hline 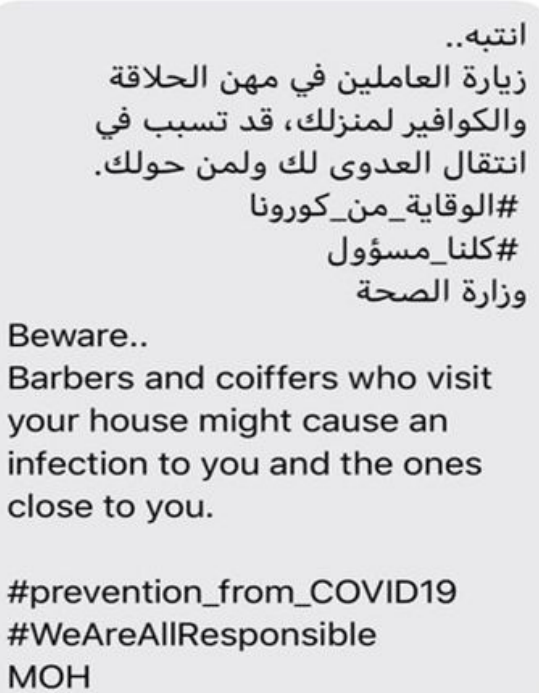 & 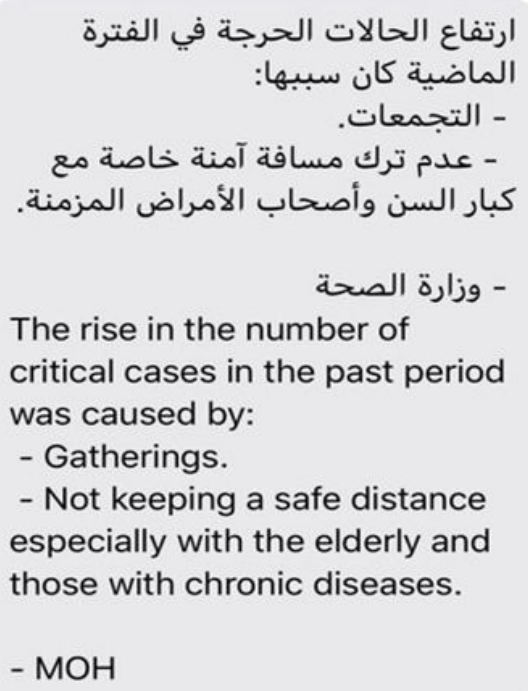 & 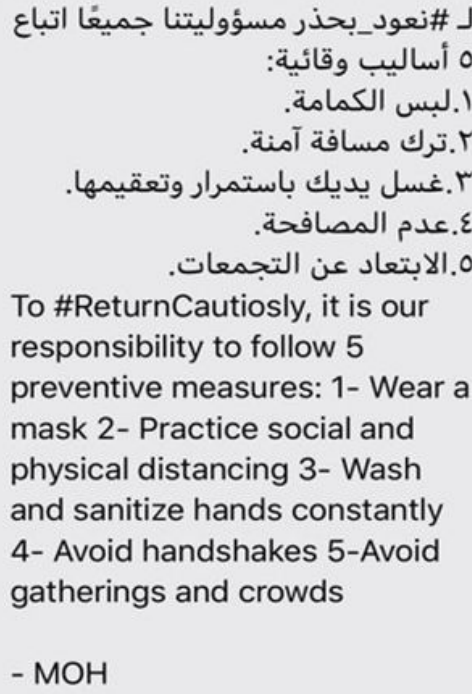 \\
\hline
\end{tabular}

\section{Figure 2}

MOH's Current Messaging Campaign 
Phase 1

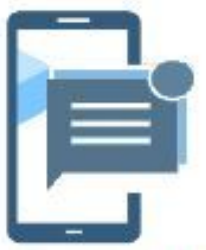

FBM-Based Awareness Messaging Campaign (Motivation, ability and triggers)

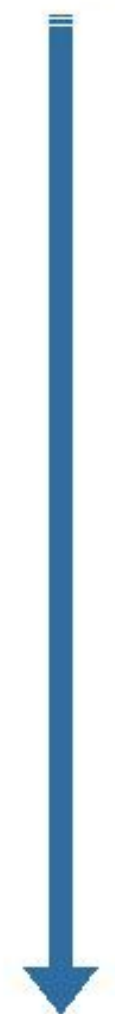

Phase 2

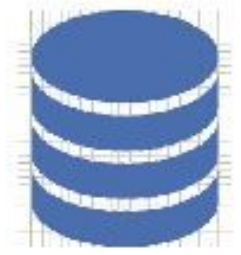

COVID-19 clinical data from health care providers

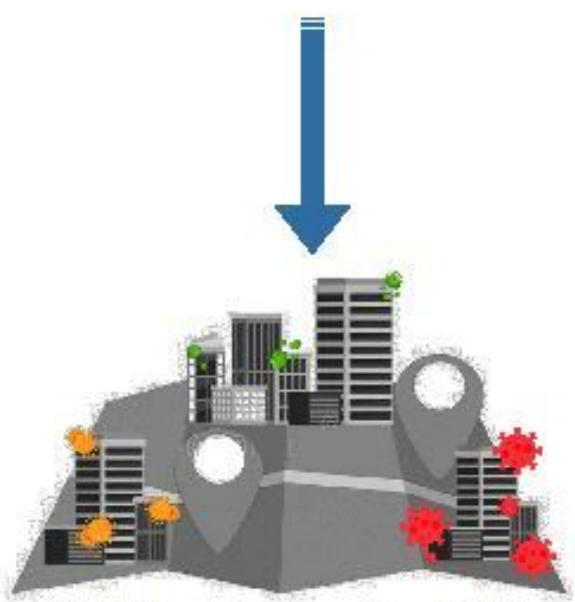

COVID-19 localized map for district profiling: 1- High infection (red)

2- Medium infection (amber)

3- Low infection (green)

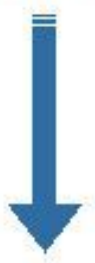

\section{Awareness Messages customized to each district}
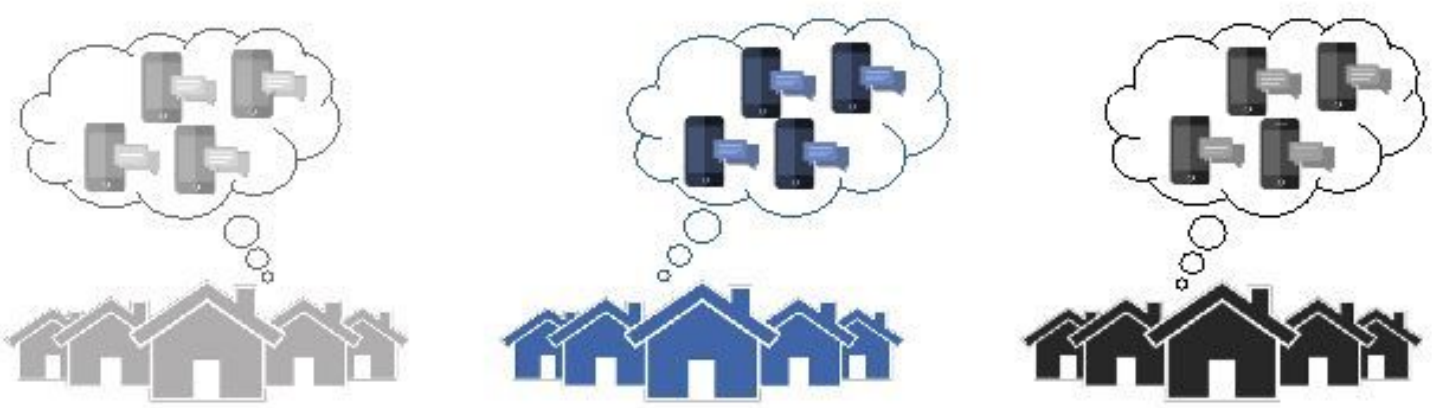

\section{Figure 3}

The main phases toward a customized awareness messaging campaign 
High Motivation

\section{Low Motivation Triggers Fail Here}

\section{Low Ability}

\section{Figure 4}

Messaging Groups Based on FBM
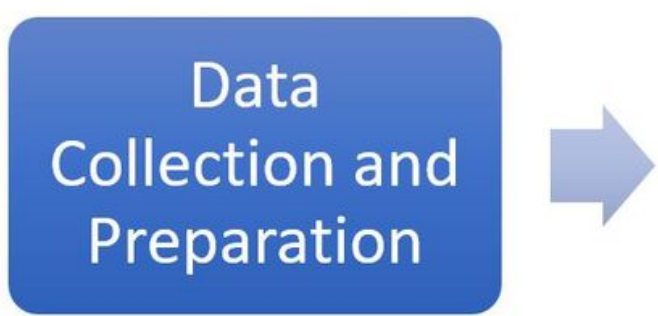

$\begin{array}{cc}\text { Facilitator Trigger } & \text { Signals Trigger } \\ \text { (Ability-Simplicity Messaging Group) } & \text { (Reminder Messaging Group) }\end{array}$

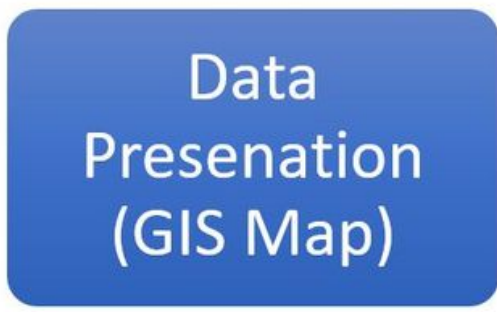

\section{High Ability}

\section{Sparks Trigger} \\ (Hope/Fear Motivator Messaging \\ Group)
}

$\begin{array}{lc}\text { Facilitator Trigger } & \text { Signals Trigger } \\ \text { (Reminder Messaging Group) }\end{array}$

$\begin{array}{lc}\text { Facilitator Trigger } & \text { Signals Trigger } \\ \text {-Simplicity Messaging Group) } & \text { (Reminder Messaging Group) }\end{array}$

Figure 5

The Technical Steps of Phase 2 


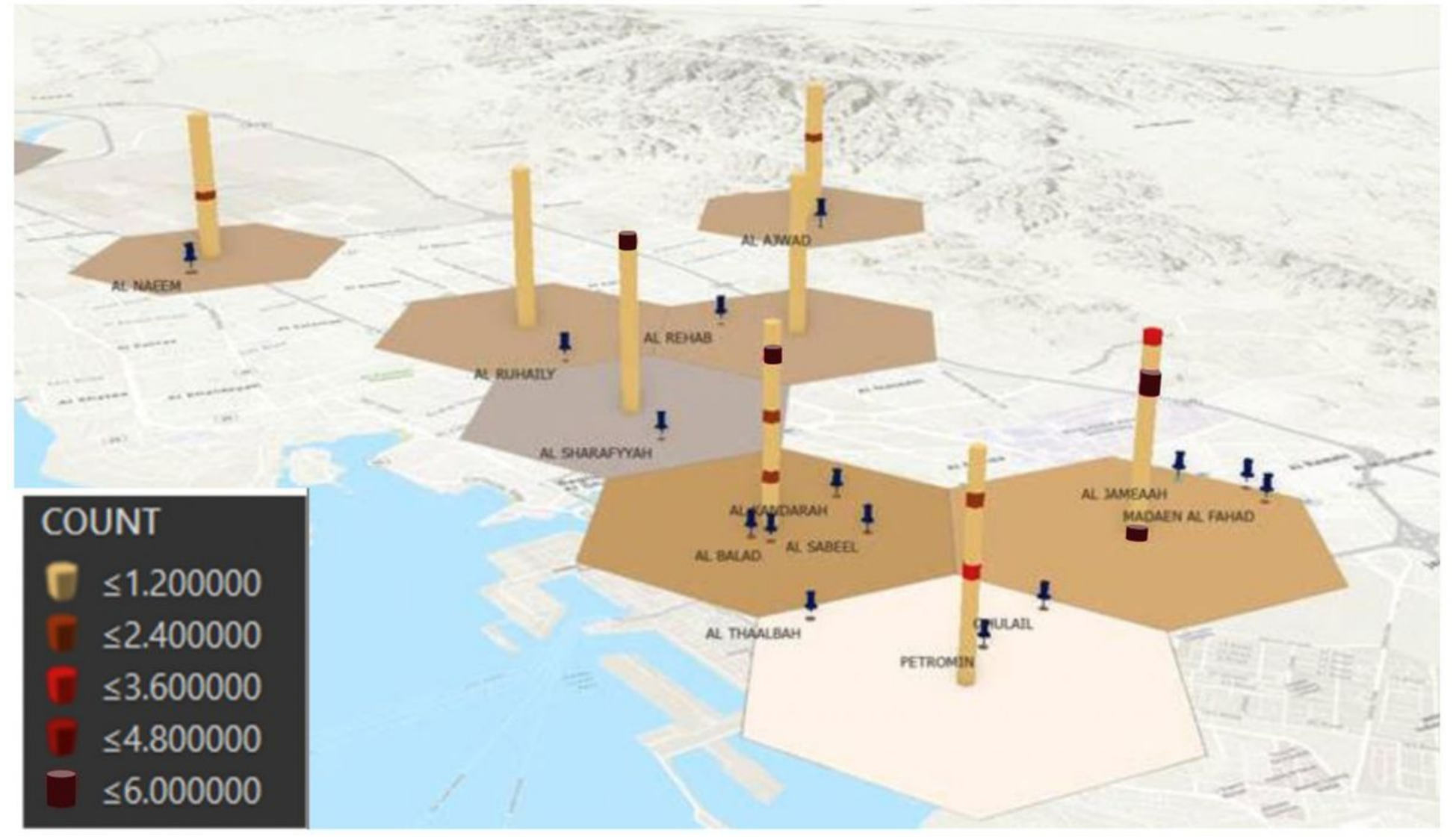

Figure 6

Space-Time Cube of COVID-19 Patients - City of Jeddah, SA

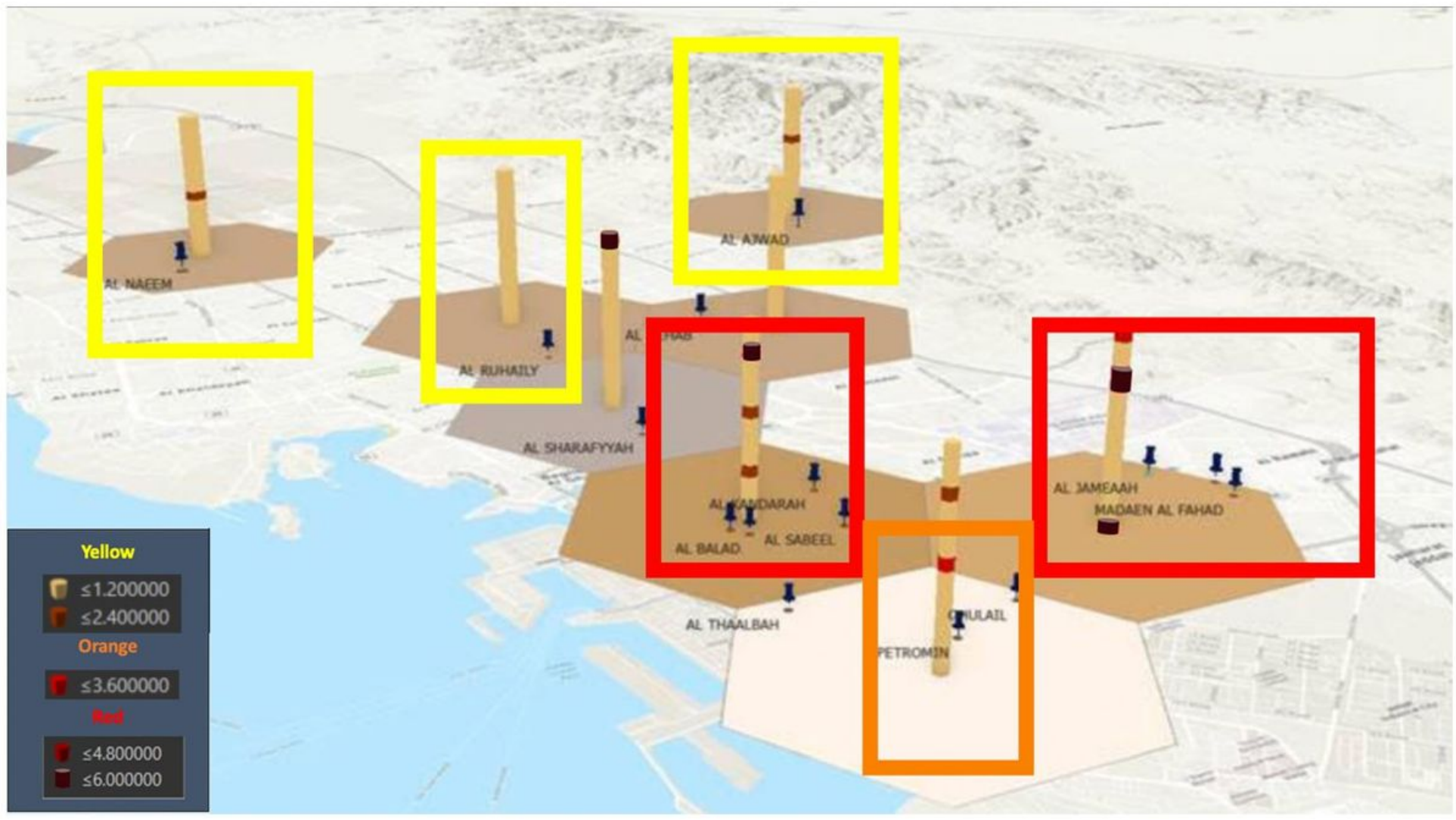


Figure 7

User Modeling Based on the District

\section{Supplementary Files}

This is a list of supplementary files associated with this preprint. Click to download.

- renamedeff17.pdf 